\title{
A SPURIOUS BROWNIAN MOTION
}

\author{
CLYDE D. HARDIN, JR.
}

\begin{abstract}
There exists an $\mathbf{R}^{d}$-valued mean-zero Gaussian process, all of whose projections agree with the projections of standard Brownian motion, yet which is not standard Brownian motion.
\end{abstract}

Suppose that $\left\{W(t)=\left(W_{1}(t), \ldots, W_{d}(t)\right): t \geqslant 0\right\}$ is an $\mathbf{R}^{d}$-valued mean-zero Gaussian process such that all projections $Y(t)=\sum_{j=1}^{d} \lambda_{j} W_{j}(t)$ behave as if $\{W(t)\}$ were standard Brownian motion in $\mathbf{R}^{d}$, i.e. $E Y(t) Y(s)=\left(\Sigma \lambda_{j}^{2}\right) \min (t, s)$. Is $\{W(t)\}$ in fact standard Brownian motion in $\mathbf{R}^{d}$ ?

Questions of this sort arise naturally when one tries to prove multidimensional analogs of some one-dimensional results by using projection methods (see, e.g., [1]).

Clearly each component of $\{W(t)\}$ is standard (one-dimensional) Brownian motion. Also, one can show that under certain "reasonable" additional hypotheses (if $\{W(t)\}$ is a martingale, for instance) that the answer to our question is yes. The following example shows that in general the (perhaps surprising) answer is no.

Let $B_{1}(t)$ and $B_{2}(t)$ be two independent standard Brownian motions. Set

$$
W_{1}(t)=B_{1}(2 t / 3)-B_{2}(t / 3) \text { and } W_{2}(t)=B_{1}(t / 3)+B_{2}(2 t / 3) .
$$

By checking covariances the reader may verify that $W(t)=\left(W_{1}(t), W_{2}(t)\right)$ has the desired properties. Yet, $\left\{W_{1}(t)\right\}$ and $\left\{W_{2}(t)\right\}$ are not independent processes, so $\{W(t)\}$ is not standard Brownian motion in $\mathbf{R}^{2}$.

ACKnowledgement. The author thanks Professor T. Kurtz for posing the question.

\section{REFERENCES}

1. T. G. Kurtz, The central limit theorem for Markov chains, Ann. Probab. 9 (1981), 557-560.

Department of Mathematics, University of Wisconsin, Milwaukee, Wisconsin 53201

Received by the editors March 21, 1984.

1980 Mathematics Subject Classification. Primary 60G15, 60J75.

Key words and phrases. Brownian motion, projection. 